\title{
Effect of a 4-week Theraband Exercise with PNF Pattem on Improving Mobility, Balance and Fear of Fall in Community-Dwelling Elderly
}

\author{
Wong YH Candace $\cdot$ Cheung KW Kennis $\cdot$ Ko YC Evelyn • Tse HC Jeffrey • \\ Law YL Margaret $\cdot$ Seong-Soo Hwang ${ }^{1 \dagger} \cdot$ Ngai PC Shirley $^{2}$ \\ St. James' Settlement, Hong Kong \\ 'Dept. of Physical Therapy, Shingu College, South Korea \\ ${ }^{2}$ Dept. of Rehabilitation Sciences, The Hong Kong Polytechnic University, Hong Kong
}

Received: July 15, 2017 / Revised: July 28, 2017 / Accepted: August 21, 2017

(c) 2017 J Korean Soc Phys Med

\section{| Abstract |}

PURPOSE: Proprioceptive Neuromuscular Facilitation (PNF) has been shown to improve body function and activity/participation in people with functional dysfunctions. This study evaluates if active exercise using theraband in PNF pattern may induce similar benefits as exercise using manual PNF pattern performed by physiotherapists on promoting mobility, balance and fear of fall in community-dwelling elderly.

METHODS: Twenty-three community-dwelling elderly with independent activities of daily living were recruited and randomly allocated into either PNF group - exercise in PNF pattern by trained physiotherapists or Theraband-PNF (T-PNF) group - exercise using theraband in PNF pattern, for an hour, twice weekly for 4 weeks. Functional outcomes such as Timed Up and Go test, Elderly Mobility Scale, Berg

†Corresponding Author : sshwang117@gmail.com This is an Open Access article distributed under the terms of the Creative Commons Attribution Non-Commercial License (http://creativecommons.org/licenses/by-nc/3.0) which permits unrestricted non-commercial use, distribution, and reproduction in any medium, provided the original work is properly cited. balance scale, functional reach and subjective measures including fear of fall (FOF) scale, bodily pain in visual analogue scale were measured pre and post-program.

RESULTS: Twenty-one participants completed the program. PNF group demonstrated significant within-group improvements in all subjective measures and objective measures. Similar within-group improvements were demonstrated in all outcome measures except FOFS in T-PNF group. However, no between-group differences were found in any of the outcome measures.

CONCLUSION: Comparable improvements in functional outcomes in community dwelling elderly were demonstrated in both groups. As manual PNF exercise traditionally need clinicians' contact and feedback on patient which limit the training to be carried out extensively in community setting. The current findings suggest that exercise using theraband in PNF pattern is feasible to be adopted as self-practice exercise for community-dwelling elderly to induce beneficial effects on functional outcomes.

Key Words: Elderly, PNF, Theraband exercise 


\section{Introduction}

Aging is defined as a gradual decline in body's ability that responds to the environment. Muscle weakness, decrease in muscle mass, reduction in sensory information processing, decrease in flexibility, balance and coordination dysfunction are common physical dysfunctions reported in people aged over sixties. Previous studies reported that muscle weakness (Pijnappels et al., 2008), dislocation and velocity of the center of mass (Melzer et al., 2004; Rubenstein, 2006), gait deficits (Rubenstein, 2006) increased incidence of falls in elderly. Other studies suggested that aging associated decreased torque and power production may increase co-activation around knee and ankle (Pijnappels et al., 2008; Pereira and Goncalves, 2011a) thereby reducing walking speed and further increased risk of fall in elderly (Lee and Kerrigan, 1999; Pereira and Goncalves, 2011b). Falls, among all external causes of morbidity and mortality, was the major reason of admission in Hong Kong in 2011-2012 and that the number was about 5 times higher than the second external cause (i.e. Exposure to inanimate mechanical forces) contributing to a large portion medical expenditure annually.

Proprioceptive Neuromuscular Facilitation (PNF) was first introduced by Dr. Kabat in early 1940s. Proprioceptive means having to do with any of the sensory receptors that give information concerning movement and position of the body; Neuromuscular means involving the nerves and muscles and Facilitation means making easier (Adler et al., 2008). The patterns of PNF exercises have a spiral, diagonal direction which emphasis more on the functional training (Kofotolis and Kellis, 2006). Through the therapist-patient interaction, therapists' hands on facilitation become an important resource to patients for motor relearning. Recent studies suggest that adopting PNF as an exercise training protocol could improve coordination, proprioception, muscle strength, physical-functional status, balance (Klein et al., 2002; Pereira and Goncalves, 2012), mobility and gait (Lee and Kerrigan, 1999) especially in elderly.

Traditionally, PNF is a manual interaction technique which needs therapists' contact and feedback on patient. Resistance is given manually by therapist throughout the PNF action and therefore one to one therapy is needed. However, such interaction may limit the training to be carried out extensively in community setting to benefit the elderly population. As an alternative, exercise program with theraband exercise in PNF pattern is introduced which also provides a certain amount of resistance throughout PNF pattern. Some researchers use elastic band for knee joint dysfunction (Park et al., 2017). However, there is limited study investigating whether or not theraband exercise in PNF pattern is as effective as manual PNF technique in inducing the aforementioned therapeutic benefits. Thus, this study aims to examine the effects of a 4-week PNF exercise program on promoting mobility, balance and self-perceived level of fear of fall in community-dwelling and to compare whether or not program adopting theraband exercise in PNF pattern is as effective as manual PNF technique by trained therapists.

\section{Methods}

\section{Participants}

Elderly aged 65 years or above, with Modified Functional Ability Category (MFAC) with category 6 or above, were recruited from the community. All participants were screened by medical professional to ensure that they could follow the instruction for performing PNF protocol. Those who have uncontrolled hypertension under medication (e.g. systolic blood pressure $>150 \mathrm{mmHg}$ or diastolic blood pressure $>90 \mathrm{mmHg}$ ), fracture in recent 6 months, active Cancer, history of stroke or any neurological disease and severe visual or hearing impairment were excluded. 


\section{Study Design}

The study was conducted between November 2013 and May 2014. Ethical approval was obtained from St James Settlement Medical ethical board. Study details were explained with written informed consent obtained prior to the participation of the study.

\section{Outcome Measures}

Pre and post-program assessments, included subjective and objective outcome measures, were conducted one week before and after the program.

\section{1) Subjective Outcome Measures}

(1) Fear of Fall Scale (FOFS) is a reliable and valid subjective measurement (Kuo and Nitz, 2011) which assesses fall risks (Austin et al., 2007) and predicts recurrent falls in elderly (Friedman et al., 2002). It includes 10 common daily activities with a reported score ranged from $0-10$, where " 0 " indicates "NO fear" and " 10 " reflects "Extremely fear", for each activity. The higher score indicates the higher possibility of fall risks.

(2) Numeric Pain Scale (NRS-11) is an 11-point self-reporting scale for assessing pain with scale ranging from 0-10. 0 indicates no pain; 1-3 represents mild pain; 4-6 represents moderate pain and 7-10 indicates severe pain.

\section{2) Objective functional outcomes}

Mobility was assessed by Timed up and Go test (TUGT) and Elderly mobility Scores (EMS) while balance was evaluated by Berg balance scale (BBS) and functional reach (FR).

(1) Timed up \& Go test (TUGT) is commonly used to evaluate functional mobility and risk of fall in elderly (Herman et al., 2011) where the participant was instructed to raise from a standardized armchair and walk 3 meters away and turn around, return back to the chair and sit down. A timed performance of less than 10 seconds reflects normal mobility level of elderly (Podsiadlo and Richardson, 1991).
(2) Elderly mobility scale (EMS) is a standardized validated and reliable scale for assessing mobility of frail elderly (Smith, 1994; Prosser et al., 1997; Spilg et al., 2001; Yu, 2007). The scale assesses 7 dimensions of functional performance which are intrinsic skills that permit the performance of complex activities of daily living.

(3) Berg balance scale (BBS) is a well-established valid and reliable assessment tool (Berg et al., 1992) with 14 items of common daily activities to evaluate performance of functional balance (Berg et al., 1989) with good sensitivity and specificity (Thorbahn and Newton, 1996, Harada et al., 1995). Each item ranges from 0 to 4 points with a total score of 14 items ranges from 0 to 56 . A range of 41-56 indicates low risk; a range of 21-40 indicates medium risk whereas a range of $0-20$ implies poor balance with high risk.

(4) Functional Reach was administered in standing where the participant was instructed to stand next to, but not touching, a wall with the arm, closer to the wall, being positioned at 90 degrees of shoulder flexion with a closed fist. The participant was instructed to reach forward without taking a step forward. The difference between the start and end position of the $3^{\text {rd }}$ metacarpal of the closed fist was recorded. The test was repeated for three trials using the result of the best attempt for analysis (Katz-Leurer et al., 2009).

\section{Exercise Programs}

Eligible participants were randomly allocated to either PNF group - exercise in PNF pattern by trained physiotherapists or Theraband PNF (T-PNF) group exercise using theraband exercise in PNF pattern. Participants in both PNF group and T-PNF group had 1-hour training session of exercise activities, twice weekly conducted in an indoor exercise center supervised by center's physiotherapists with qualification of PNF level $3 \mathrm{a}$ or above. The whole program lasted for 4 weeks $(8$ sessions in total). Participants, with bodily pain, were 
Table 1. Detailed protocol used in PNF and T-PNF group

\begin{tabular}{|c|c|c|c|c|}
\hline & $\begin{array}{l}\text { Position of } \\
\text { Patients }\end{array}$ & Pattern used & $\begin{array}{l}\text { Technique / Basic } \\
\text { Principle used }\end{array}$ & Duration \\
\hline \multirow{6}{*}{$\begin{array}{l}\text { PNF } \\
\text { group }\end{array}$} & Supine & Bridging & Replication; COI & 5 minutes \\
\hline & Supine & $\begin{array}{l}\text { LE: Flex/Add/ER with } \\
\text { Knee Flex }\end{array}$ & $\begin{array}{l}\text { RI/Resistance/ } \\
\text { Traction }\end{array}$ & $\begin{array}{l}5 \text { minutes for both } \\
\text { legs }\end{array}$ \\
\hline & Supine & $\begin{array}{l}\text { LE: Ext/Abd/IR with } \\
\text { Knee Ext }\end{array}$ & $\begin{array}{l}\text { RI/Resistance/ } \\
\text { Approximation }\end{array}$ & $\begin{array}{l}5 \text { minutes for both } \\
\text { legs }\end{array}$ \\
\hline & Sitting & Sit to stand & $\begin{array}{l}\text { Approximation, COI, } \\
\text { SR }\end{array}$ & 5 minutes \\
\hline & Standing & One leg emphasis & $\begin{array}{l}\text { Approximation/ } \\
\text { Resistance }\end{array}$ & 5 minutes \\
\hline & Standing & Forward Gait & $\begin{array}{l}\text { Approximation/ } \\
\text { Resistance }\end{array}$ & 5 minutes \\
\hline \multirow{6}{*}{$\begin{array}{l}\text { T-PNF } \\
\text { group }\end{array}$} & Supine & Bridging & - & 5 minutes \\
\hline & Side lying & $\begin{array}{l}\text { LE: Ext/Abd/IR with } \\
\text { Knee Ext }\end{array}$ & - & $\begin{array}{l}5 \text { minutes for both } \\
\text { legs }\end{array}$ \\
\hline & Supine & $\begin{array}{l}\text { LE: Flex/Add/ER with } \\
\text { Knee Flex }\end{array}$ & - & $\begin{array}{l}5 \text { minutes for both } \\
\text { legs }\end{array}$ \\
\hline & Sitting & Sit to stand & - & 5 minutes \\
\hline & Standing & One leg emphasis & - & 5 minutes \\
\hline & Standing & Forward Gait & - & 5 minutes \\
\hline
\end{tabular}

Note. LE=lower extremity; Flex=Flexion; Add=Adduction; ER=External Rotation; Ext=Extension; Abd=Abduction; $\mathrm{IR}=$ Internal Rotation

encouraged to exercise within their available joint range. In each session, vital signs such as blood pressure, heart rate, body temperature and resting level of oxygen saturation $\left(\mathrm{SpO}_{2}\right)$ were measured before exercise to assure stable physical status. Standardized warm up and cool down exercise including leisure walking for 5 minutes; stretching of bilateral quadriceps, hamstrings and calves in standardized position with 1 minute per muscle group; and active ankle dorsiflexion and plantarflexion for 10 times (Table 1).

\section{1) PNF group}

Six PNF patterns or functional activities were chosen. In specific, PNF patterns that could improve trunk stability and power of lower limb were chosen. Majority of the chosen patterns were closed-chain movements which simulated functional movement during walking while the chosen opened-chain movement was pattern involving flexion of hip and knee and extension of hip and knee which emphasized strengthening of the muscle power of lower limb during walking. Different body positions from lower level position in supine lying to higher level of position in standing were adopted. Participants learnt and performed exercise in a more stable and easy position first, then progressed to a more functional position. Apart from following exercise pattern, PNF basic procedures and principles based on the philosophy, tactile stimulation, manual contact, audio input, correct timing, appropriate resistance, approximation or traction were applied to the participant during the exercise. Details of the exercise program were listed in Table 1 with movements and patterns demonstrated in Fig. 1. 


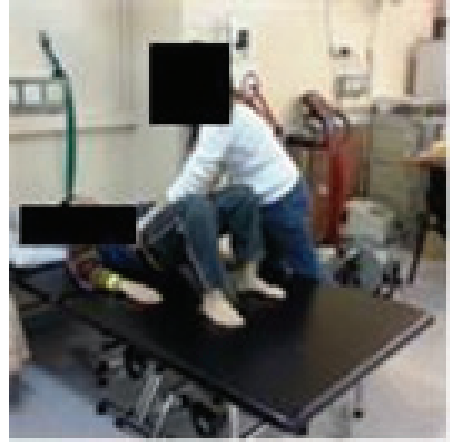

(1) Bridging

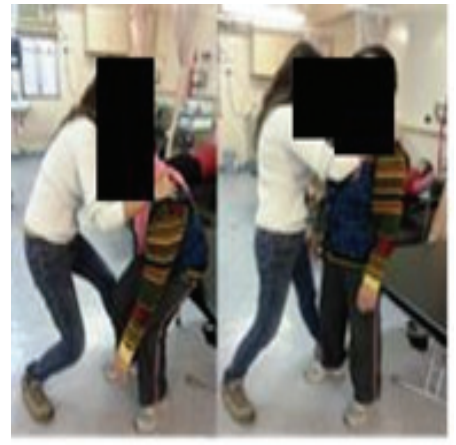

(4) Sit-to-stand

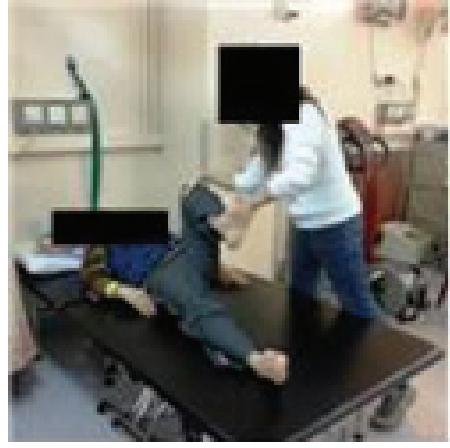

(2) LE: Flex/Add/ER with Knee Flex

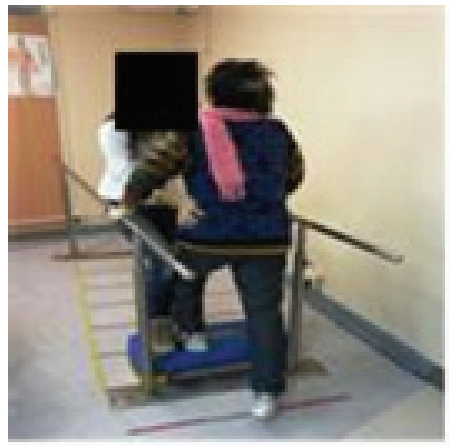

(5) Gait training: One leg emphasis

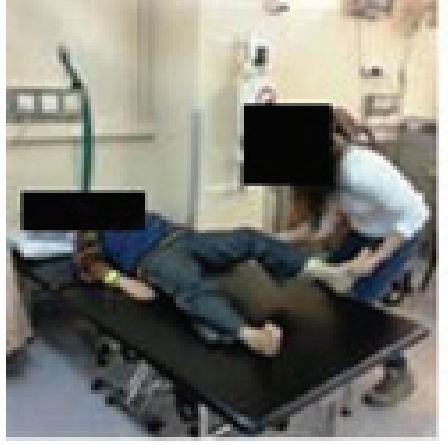

(3) LE: Ext/Abd/IR with knee ext

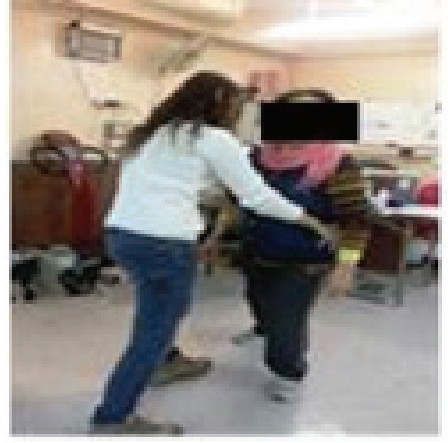

(6) Gait training: Forward Gait

Note. LE=lower extremity; Flex=Flexion; Add=Adduction; ER=External Rotation; Ext=Extension; Abd=Abduction; $\mathrm{IR}=$ Internal Rotation

Fig. 1. Detailed PNF pattern and functional activities in PNF group

\section{2) T-PNF group}

PNF pattern and functional activities adopted in this group were identical to those used in PNF group. The main differences were that theraband and anti-gravity position were used to replace the manual resistance by therapist. Participants were taught to perform exercise in PNF pattern and specific direction by therapists by 5 times without any resistance given. Then they started to perform the exercise by themselves. Details of the exercise program were listed in Table 1 with T-PNF patterns and movements demonstrated in Fig. 2.

\section{Statistical Analysis}

All data were presented as mean \pm standard deviation (SD). Demographic and baseline data between two groups were compared by independent t-test. Paired t-test was used to examine within group change measured pre and post program in each group while independent t-test was used to examine the between group differences. Data were analyzed using SPSS (version 23, IBM Corporation, USA). Statistical significance was set at .05.

\section{Results}

Twenty-three participants were recruited in the study from November 2013 to January 2014 at St James Settlement Central-Western District Center. Of these, twenty-one participants completed the program. In PNF group, 1 out of 13 participants quitted due to the persistently 


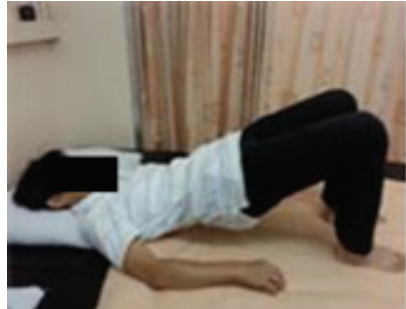

(1) Bridging

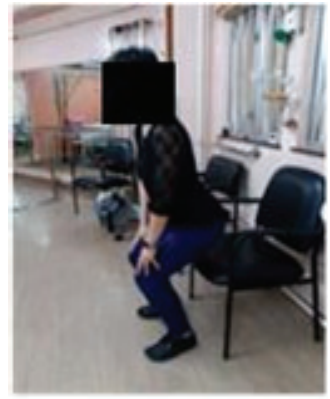

(4) Sit-to-stand

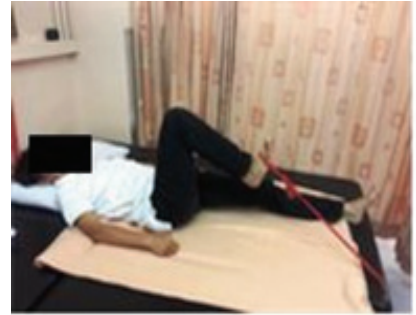

(2) LE: Flex/Add/ER with Knee Flex

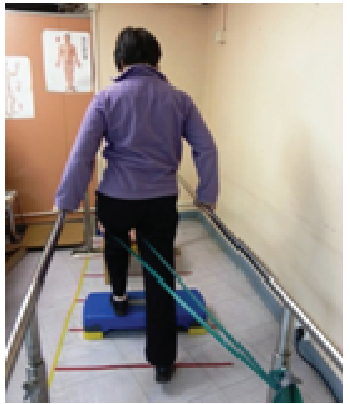

(5) Gait training: One leg emphasis

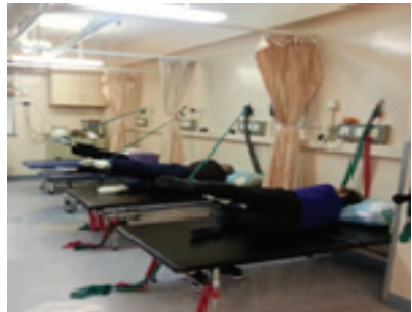

(3) LE: Ext/Abd/IR with knee ext

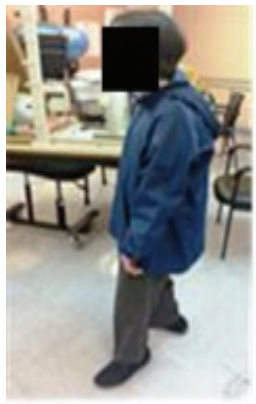

(6) Gait training: Forward Gait

Note. LE=lower extremity; Flex=Flexion; Add=Adduction; ER=External Rotation; Ext=Extension; Abd=Abduction; $\mathrm{IR}=$ Internal Rotation

Fig. 2. Detailed PNF pattern and functional activities in T-PNF group

high blood pressure measured before exercise sessions while in T-PNF group, 1 out of 10 participants did not complete post-program assessment due to hospitalization contributing to an overall attrition rate of $8.7 \%$ in this study. Mean age of participants in PNF and T-PNF group were $78.4 \pm .6 .3$ and $75.6 \pm 8.7$ years old respectively. No significant between-group differences were found in the baseline measurement of FOFS, TUGT, EMS and BBS score. Details of the demographic data were shown in Table 2 .

Table 2. Characteristics and baseline parameters of participants

\begin{tabular}{lccc}
\hline & PNF $(\mathrm{n}=12)$ & T-PNF $(\mathrm{n}=9)$ & P value \\
\hline Age, year & $78.4 \pm 6.3$ & $75.6 \pm 8.7$ & .39 \\
Gender (F, \%) & $91 \%$ & $67 \%$ & .27 \\
FOF, unit & $43.8 \pm 17.1$ & $42.2 \pm 17.9$ & .85 \\
Pain VAS, unit & $4.9 \pm 1.8$ & $5.1 \pm 3.7$ & .89 \\
EMS, unit & $18.9 \pm 1.4$ & $18.4 \pm 1.7$ & .50 \\
TUGT, sec & $11.0 \pm 2.3$ & $12.3 \pm 8.8$ & .67 \\
FR, cm & $21.2 \pm 5.8$ & $20.1 \pm 6.8$ & .70 \\
BBS, unit & $49.9 \pm 3.2$ & $47.8 \pm 6.4$ & .38 \\
\hline
\end{tabular}

Data are presented as mean \pm standard deviation (SD) unless otherwise indicated. $\mathrm{PNF}=$ exercise in PNF pattern conducted by therapists; T-PNF=exercise using theraband in PNF pattern; FOF=Fear of Fall; VAS=pain measured in visual analogue scale; EMS=Elderly Mobility Scale; TUGT=Timed-up-and-go test; FR=Functional Reach; BBS=Berg Balance Scale. 
Table 3. Comparison of outcome measures in PNF and T-PNF group

\begin{tabular}{|c|c|c|c|c|c|c|c|}
\hline & \multirow{2}{*}{\multicolumn{2}{|c|}{ PNF $(n=12)$}} & \multirow{2}{*}{\multicolumn{2}{|c|}{ T-PNF $(n=9)$}} & \multicolumn{3}{|c|}{$\mathrm{P}$ value } \\
\hline & & & & & \multicolumn{2}{|c|}{ Within group } & \multirow{2}{*}{$\begin{array}{c}\text { Between } \\
\text { group }\end{array}$} \\
\hline & Pre Program & Post Program & Pre Program & Post Program & $\mathrm{PNF}$ & T-PNF & \\
\hline FOF, unit & $43.8 \pm 17.1$ & $23.7 \pm 14.6$ & $42.2 \pm 17.9$ & $35.2 \pm 15.4$ & $.014 *$ & .07 & .11 \\
\hline VAS, unit & $4.9 \pm 1.8$ & $2.9 \pm 2.7$ & $5.1 \pm 3.7$ & $3.4 \pm 3.5$ & $.049 *$ & .07 & .79 \\
\hline EMS, unit & $18.9 \pm 1.4$ & $19.7 \pm .8$ & $18.4 \pm 1.7$ & $19.8 \pm .7$ & $.043^{*}$ & $.02 *$ & .31 \\
\hline TUGT, sec & $11.0 \pm 2.3$ & $8.1 \pm 2.2$ & $12.3 \pm 8.8$ & $9.9 \pm 6.7$ & $<.001 * *$ & $.02 *$ & .63 \\
\hline $\mathrm{FR}, \mathrm{cm}$ & $21.2 \pm 5.8$ & $26.7 \pm 3.3$ & $20.1 \pm 6.8$ & $28.9 \pm 5.9$ & $.016^{*}$ & $.009^{* *}$ & .31 \\
\hline BBS, unit & $49.9 \pm 3.2$ & $53.6 \pm 1.9$ & $47.8 \pm 6.4$ & $51.8 \pm 6.3$ & $.001^{* *}$ & $.006^{* *}$ & .81 \\
\hline
\end{tabular}

Data are presented as mean \pm standard deviation (SD) unless otherwise indicated. PNF=exercise in PNF pattern conducted by therapists; $\mathrm{T}-\mathrm{PNF}=$ exercise using theraband in $\mathrm{PNF}$ pattern; $\mathrm{FOF}=\mathrm{Fear}$ of Fall; VAS=pain measured in visual analogue scale; EMS=Elderly Mobility Scale; TUGT=Timed-up-and-go test; FR=Functional Reach; BBS=Berg Balance Scale; * denotes significant within-group differences at $\mathrm{p}<.05$. ${ }^{* *}$ denotes significant within-group differences at $\mathrm{p}<.01$; \#denotes significant between group between at $\mathrm{p}<.05$.

Both PNF group and T-PNF group demonstrated significant within group improvement in all of outcomes measures after the 4-week program (Table 3) except FOFS in T-PNF group. No between group differences were found in any of the outcome measures (Table 3).

\section{Discussion}

This study demonstrated that a 4-week program of PNF and T-PNF training could improve functional outcomes such as mobility and balance in community dwelling elderly. Improvement in subjective outcomes such as VAS and FOFS, however, was only observed in PNF group.

Generally there are many elastic band and theraband exercise without PNF pattern for the strengthening (Park and Lee, 2016) and improving postural control (Kim et al., 2016). Previous studies reported that PNF training improves muscle endurance (Kofotolis et al., 2005), muscle power (Klein et al., 2002) and selected functional tasks (Klein et al., 2002) such as sit-to-stand, balance, mobility for those elderly after PNF training. Such improvement could probably be related to the increase in cross-sectional areas of muscles (Kofotolis et al., 2005). In accord with previous studies, this present study also showed a consistent improvement in functional outcomes.

Both groups demonstrated significant improvement on TUGT. Gait training is one of the main focuses in PNF concept. Its effects on promoting gait performance, as reflected by changes in walking speed and cadence, have been shown in people with various neurological and musculoskeletal conditions such as Parkinson's disease (Mirek et al., 2003); Stroke (Wang, 1994) and amputation (Yiğiter et al., 2002). All these may possibly explain the improvement observed.

Other than objective improvement, participants in PNF group also revealed a positive subjective improvement on reducing fall risks and level of pain. Both subjective and objective findings could be inter-related. The better performance in functional outcomes such as mobility and balance may result in more functional independence while attaining stability when performing mobility and daily activities. All these may increase their self confidence in 
performing functional tasks and reduce their self-perceived fear of falling and immobile.

However, there was no subjective improvement shown in T-PNF group after the program. There were difference between T-PNF group and PNF group. First, there was manual contact from the therapists to the participant. Through this, tailor-made resistance could be added to the participants according to their ability. Second, approximation was added to the participants during Sit-to-Stand training which aimed to improve the stability during standing by increasing sensory input through approximation. Moreover, traction during lower limb patterns especially when flexion pattern was added to the limbs to promote mobility and reduce pain if pain was from joint compression. All these were only applied in PNF group but not T-PNF group which may explain such insignificant difference observed in T-PNF group.

As aforementioned, this study aims to compare the effectiveness of exercise using theraband in PNF pattern with manual PNF applied by therapists. In community, manual PNF therapy is difficult to execute among large elderly population as it is labor intensive. Our findings demonstrated that both groups also improved objective outcomes with NO between-group difference. These results suggest that theraband exercise in PNF pattern can be an alternative exercise therapy that could be carried out in community. Large aging population with high fall risk is an essential issue in society which causes heavy government medical burden. If elderly could perform theraband exercise using PNF pattern as a home exercise regularly, they could be benefited from exercise specifically improving the mobility and balance further decreasing risk of fall.

However, with better results gained subjectively in the PNF group than the T-PNF group, we still believe that T-PNF group might not be able to gain as maximal as from manual application. No detailed adjustment on resistance could be made within the available range of motion according to the performance from participants in T-PNF group. Furthermore, NO manual contact was delivered in T-PNF group that was vital in sensory input to stimulate the target muscles. Furthermore, without manual contact, traction and approximation could not be applied which would decrease proprioceptive input to the joints involved thereby affecting the training effect on mobility and stability as well as pain reduction. Velocity normalization would be minimized in T-PNF group with no manual facilitation. All these differences between groups possibly explained the outcomes variations after intervention. In addition, small sample size in this study may also contribute a potential bias in the results. Hence, further study with larger sample size in warranted.

\section{Conclusion}

Comparable improvements in functional outcomes in community dwelling elderly were demonstrated in both groups. As manual PNF exercise traditionally need clinicians' contact and feedback on patient which limit the training to be carried out extensively in community setting. The current findings suggest that exercise using theraband in PNF pattern is feasible to be adopted as self-practice exercise for community dwelling elderly to induce beneficial effects on functional outcomes.

\section{Acknowledgements}

This study was funded from the social welfare development fund by the Social Welfare Department of the HKSAR government to St. James' Settlement, Hong Kong. Authors would like to thank the International Proprioceptive Neuromuscular Facilitation Association- Hong Kong, China for all technical support. 


\section{References}

Adler S, Beckers D, Buck M. PNF in practice: an illustrated guide $\left(3^{\text {rd }}\right.$ ed). Germany. Springer Medizin Verlag. 2008.

Austin N, Devine A, Dick I, et al. Fear of falling in older woman: a longitudinal study in incidence, persistence and predictors. J Am Geriatr Soc. 2007;55:1598-603.

Berg KO, Wood-Dauphinée S, Williams JI, et al. Measuring balance in the elderly: preliminary development of an instrument. Physiotherapy Canada. 1989;41: 304-11.

Berg KO, Wood-Dauphinée SL, Williams JI, et al. Measuring balance in the elderly: validation of an instrument. Can J Public Health. 1992;83:S7-11.

Friedman SM, Munoz B, West SK, et al. Falls and fear of falling: which comes first? a longitudinal prediction model suggests strategies for primary and secondary prevention. J Am Geriatr Soc. 2002;50:1329-35.

Harada U, Chiu V, Damon-Rodriguez J, et al. Screening for balance and mobility impairment in elderly individual living in residential care facilities. Phys Ther. 1995; 75:462-9.

Herman T, Giladi N, Hausdorff JM. Properties of the 'timed up and go' test: more than meets the eye. Gerontology. 2011;57:203-10.

Katz-Leurer M, Fisher I, Neeb M, et al. Reliability and validity of the modified functional reach test at the sub-acute stage of post-stroke. Disabil Rehabil. 2009;31(3): 243-8.

Kim DS, Choi IS, Kim SY. The impact of lower extremity strengthening exercise with step box and elastic band on balance ability and lower extremity strength in community-living elderly individuals. J Korean Soc Phys Med. 2016;11(1):11-21.

Klein DA, Stone WJ, Philips WT, et al. PNF training and physical function in assisted-living older adults. J Aging Phys Act. 2002;10:476-88.
Kofotolis N, Kellis E. Effects of two 4-week proprioceptive neuromuscular facilitation programs on muscle endurance, flexibility, and functional performance in women with chronic low back pain. Phys Ther. 2006;86:1001-12.

Kofotolis N, Vrabas IS, Vamvakoudis K, et al. Proprioceptive neuromuscular facilitation training induced alterations in muscle fiber type and cross sectional area. $\mathrm{Br}$ J Sports Med. 2005;39:e11.

Kuo SL, Nitz JC. Establishment of predictive validity and reliability of a newly developed fear of falling scale in Hong Kong. Hong Kong Physiotherapy Journal. 2011;29:2-11.

Lee LW, Kerrigan DC. Identification of kinetic differences between fallers and nonfallers in the elderly. Am J Phys Med Rehabil. 1999;78:242-6.

Melzer I, Benjuya N, Kaplanski J. Postural stability in the elderly: a comparison between fallers and non-fallers. Age Ageing. 2004;33:602-7.

Mirek E, Chwała W, Longawa K, et al. Proprioceptive neuromuscular facilitation method of therapeutic rehabilitation in the treatment of patients with Parkinson disease. Neurologia i neurochirurgia polska. 2003;37(S5):89-102.

Park MC, Lee SY. Deltoid middle fiber of the isometric contraction according to elastic band and dumbbell on various shoulder abduction angle. J Korean Soc Phys Med. 2016;12(4):71-6.

Park SR, Ro HL, Namkoong S. The effects of stretching and elastic band exercises knee space distance and plantar pressure distribution during walking in young individuals with genu varum. J Korean Soc Phys Med. 2017;12(1):83-91.

Pereira M, Goncalves M. Effects of fatigue induced by prolonged gait when walking on the elderly. Human Movement. 2011b;12:242-7.

Pereira M, Goncalves M. Muscular coactivation around the knee reduces power production in elderly women. 
Arch Gerontol Geriatr. 2011a;52:317-21.

Pereira M, Goncalves M. Proprioceptive neuromuscular facilitation improves balance and knee extensors strength of older fallers. ISRN Rehabilitation. 2012: 1-7. Article ID 402612, doi:10.5402/2012/402612.

Pijnappels M, van der Burg JCE, Reeves ND, et al. Identification of elderly fallers by muscle strength measures. Eur J Appl Physiol. 2008;102:585-92.

Podsiadlo D, Richardson S. The timed Up \& Go: a test of basic functional mobility for frail elderly persons. J Am Geriatr Soc. 1991;39:142-8.

Prosser L, Canby A. Further validation of EMS for measurement of mobility of hospitalized elderly people. Clin Rehabil. 1997;11:338-4.

Rubenstein LZ. Falls in older people: epidemiology, risk factors and strategies for prevention. Age Ageing. 2006;35(S2):ii37-ii41.

Smith R. Validation and reliability of the elderly mobility scale. Physiotherapy. 1994;80:744-7.

Spilg EG, Martin BJ, Mitchell SL, et al. A comparison of mobility assessments in a geriatric day hospital. Clin Rehabil. 2001;15:296-300.

Thorbahn LDB, Newton RA. Use of the Berg Balance Test to predict falls in elderly persons. Phys Ther. 1996;76:576-85.

Wang RY. Effect of proprioceptive neuromuscular facilitation on the gait of patients with hemiplegia of long and short duration. Phys Ther. 1994;74:1108-15.

Yiğiter K, Sener G, Erbahçeci F, et al. A comparison of traditional prosthetic training versus proprioceptive neuromuscular facilitation resistive gait training with trans-femoral amputees. Prosthet and Orthot Int. 2002;26:213-7.

$\mathrm{Yu}$ MSW. Usefulness of the elderly mobility scale for classifying residential placements. Clin Rehabil. 2007;21:1114-20. 